\title{
Numerical computation of the Shock Tube Problem by means of wave digital principles
}

\author{
A. Mengel \\ Lehrstuhl für Nachrichtentechnik, Ruhr-Universität, Bochum, Germany
}

\begin{abstract}
Partial differential equations can be solved numerically by means of wave digital principles. The great advantage of this method is the simultaneous achievement of high robustness, massive parallelism full localness and high accuracy. Among others this method will be applied in order to solve the Euler-equations according to one dimension in space. Especially the so called Shock Tube Problem will be examined. The analytical solution of this problem contains two discontinuities, namely a shock and a contact discontinuity. These result in oscillations which are due to numerical integration methods of higher order. Also solutions of the Wave Digital Method contain these oscillations, contrary to what had been observed of Yuhui Zhu (2000). This behaviour is also known as Gibbs Phenomena.

The Navier-Stokes-equations, which are from a physical point of view more exactly, additionally take viscosity terms into account. This leads to smooth solutions near shocks. It will be shown that this approach leads to the suppression of the oscillations near the shock. Furthermore it will be shown that quite good results for the computation of velocity and pressure can be obtained.
\end{abstract}

\section{Introduction}

In the past Fries (1994) already solved the Shock Tube Problem by means of Wave Digital Principles. There every single Euler-equation was represented by an energy equation of a separate multidimensional (MD) Kirchhoff network. In order to avoid oscillations near the discontinuities, Fries introduced artificial terms of dissipation and heat conduction.

Whereas in this paper the Euler-equations are represented by Kirchhoff laws, which are applied to an appropriate MD Kirchhoff network. In a further step this network can be extended in a way that viscosity terms are taken into account.

Correspondence to: A. Mengel (mengel@nt.rub.de)
Concerning the differential equations this corresponds to the transition to the Navier-Stokes-equations.

\section{Equations of fluid dynamics}

Starting point for the approach presented in this paper are the equations of fluid dynamics. A special form of these has been introduced by Fettweis (2002). In the case that only one dimension in space is considered, these equations are given in the form

$$
\begin{aligned}
\mathrm{D}_{t} e_{\mathrm{c}}+\mathrm{D}_{x}\left(v e_{\mathrm{c}}\right)+v \mathrm{D}_{x} p & =v f \\
\mathrm{D}_{t} e_{\mathrm{i}}+\mathrm{D}_{x}\left(v e_{\mathrm{i}}\right)+p \mathrm{D}_{x} v & =q_{\mathrm{f}} \\
\mathrm{D}_{t} \rho+\mathrm{D}_{x}(v \rho) & =0
\end{aligned}
$$

where

$$
\mathrm{D}_{x}=\partial / \partial x, \quad \mathrm{D}_{t}=\partial / \partial t,
$$

$v=$ velocity, $\quad p=$ pressure,$\quad \rho=$ density,

$e_{\mathrm{c}}=\frac{1}{2} \rho v^{2}$ kinetic energy density,

$e_{\mathrm{i}}=$ internal (thermodynamic) energy density,

$f=$ force due to viscosity,

$q_{\mathrm{f}}=$ power density supplied to $e_{\mathrm{i}}$ by the viscosity forces.

If $f$ is chosen in a way which corresponds to Newton, Eqs. (1-3) are equivalent to the Navier-Stokes-equations. However the terms which represent heat conduction and external forces are not taken into account. Equation (1) represents the conservation of kinetic energy. This is equivalent to the equation of momentum conservation, which is normally used in fluid dynamics. However using Eq. (1) has advantages in deriving an appropriate MD Kirchhoff network.

If the terms $f$ and $q_{\mathrm{f}}$ are ignored, Eqs. (1-3) are equivalent to the Euler-equations.

In the following it is assumed that the fluid is a perfect gas. The relation between pressure and internal energy density is given by the following equation

$$
e_{\mathrm{i}}=\frac{p}{\gamma-1}, \quad 1<\gamma \leq 2, \quad \gamma=\text { isentropic coefficient. }
$$




\section{Coordinate transformation}

In order to get a MD Kirchhoff network Eqs. (1-3) have to be transformed in a suitable way. For this purpose these equations are written in dependence of vector

$\boldsymbol{x}=\left(x_{1}, x_{2}\right)^{\mathrm{T}}=\left(x, v_{0} t\right)^{\mathrm{T}}$.

After another transformation these depend on the MD time vector

$\boldsymbol{t}=\left(t_{1}, t_{2}\right)^{\mathrm{T}}$.

The transformation has to be applied in a way that the appropriate system gets causal concerning every component of $\boldsymbol{t}$. This is a necessary condition for the MD passivity of the system. A suitable transformation which is used here is given by

$\boldsymbol{x}=v_{0} \boldsymbol{H} \boldsymbol{t} \quad$ where $\quad \boldsymbol{H}=\frac{1}{\sqrt{2}}\left(\begin{array}{rr}1 & -1 \\ 1 & 1\end{array}\right)$.

In order to achieve MD causality of the system $v_{0}$ has to be chosen sufficiently large. The appropriate differential operators are resulting in

$$
\left(\begin{array}{l}
\mathrm{D}_{x_{1}} \\
\mathrm{D}_{x_{2}}
\end{array}\right)=\frac{1}{v_{0} \sqrt{2}}\left(\begin{array}{rr}
1 & -1 \\
1 & 1
\end{array}\right)\left(\begin{array}{l}
\mathrm{D}_{1} \\
\mathrm{D}_{2}
\end{array}\right)
$$

where $\quad \mathrm{D}_{1}=\partial / \partial t_{1}, \quad \mathrm{D}_{2}=\partial / \partial t_{2}$.

\section{MD Kirchhoff network}

Similar as in Fettweis (2002) using Eqs. (7) and (8) and applying some manipulations, Eqs. (1-3) can be transformed in the form

$$
\begin{aligned}
& \sum_{\kappa=1}^{2} \sqrt{L_{\kappa}} \mathrm{D}_{\kappa}\left(\sqrt{L_{\kappa}} \tilde{v}\right)+\sqrt{L}\left(\mathrm{D}_{1}-\mathrm{D}_{2}\right)(\sqrt{L} \tilde{p})=\tilde{f} \\
& \sum_{\kappa=1}^{2} \sqrt{L_{4 \kappa}} \mathrm{D}_{\kappa}\left(\sqrt{L_{4 \kappa}} \tilde{p}\right)+\sqrt{L}\left(\mathrm{D}_{1}-\mathrm{D}_{2}\right)(\sqrt{L} \tilde{v})=\frac{\tilde{q}_{\mathrm{f}}}{\tilde{p}} \\
& \sum_{\kappa=1}^{2} \sqrt{L_{0 \kappa}} \mathrm{D}_{\kappa}\left(\sqrt{L_{0 \kappa}} \tilde{\rho}\right) \\
& =0
\end{aligned}
$$

where

$$
\begin{aligned}
& \tilde{v}=\frac{v}{v_{0}} \sqrt{\frac{p}{p_{0}}}, \tilde{p}=\sqrt{\frac{p}{p_{0}}}, \tilde{\rho}=\sqrt{\frac{\rho}{\rho_{0}}}, \tilde{f}=v_{0} \sqrt{\frac{1}{2 p p_{0}}} f, \\
& \tilde{q}_{\mathrm{f}}=\frac{q_{\mathrm{f}}}{\sqrt{2} p_{0}}, \quad v_{0}>0, \quad p_{0}>0, \quad \rho_{0}>0
\end{aligned}
$$

and

$$
\begin{aligned}
L & =1, \quad L_{0 v}=1 \pm \frac{v}{v_{0}}, \quad L_{v}=\frac{v_{0}^{2} \rho}{2 p} \pm \frac{v_{0} v \rho}{2 p} \\
L_{4 v} & =\frac{e_{\mathrm{i}}}{p} \pm \frac{\left(e_{\mathrm{i}}-p\right) v}{v_{0} p}, \quad v=1,2 .
\end{aligned}
$$

Equations (9-11) can be interpreted as mesh equations of an appropriate MD Kirchhoff network. But first of all a closer look at the viscosity terms has to be taken.

\subsection{Viscosity terms}

In the case that three dimensions in space are considered, the forces due to viscosity result from

$\boldsymbol{f}=\left(\nabla^{\mathrm{T}} \boldsymbol{S}\right)^{\mathrm{T}}$ where $\nabla=\left(\mathrm{D}_{x}, \mathrm{D}_{y}, \mathrm{D}_{z}\right)^{\mathrm{T}}$.

Matrix $S$ corresponds to the so called stress tensor which is normally used in mechanics (Böhme, 1990). Due to the viscosity forces energy is dissipated which results from

$q_{\mathrm{f}}=(\boldsymbol{S} \nabla)^{\mathrm{T}} \boldsymbol{v}$.

Every component of matrix $S$ depends on the considered material behaviour. In this case the Newtonian fluid is taken into account. This approach assumes a linear dependence between mechanical stresses and gradients of velocity.

If one dimension in space is considered, the following approach is given

$f=D_{x} s_{x x} \quad$ where $s_{x x}=\eta D_{x} v$.

Thus the energy density $q_{\mathrm{f}}$ is given by

$q_{\mathrm{f}}=\eta\left(\mathrm{D}_{x} v\right)^{2}$.

Introducing another variable $w$, where

$w+\eta \mathbf{D}_{x} v=0$,

$\eta$ viscosity coefficient $>0$,

one obtains a differential equation in $w$, which is not hyperbolic in the sense that a finite propagation velocity has to be assumed. With this, the energy density $q_{\mathrm{f}}$ can also be written in dependence of $w$, where

$q_{\mathrm{f}}=\frac{w^{2}}{\eta}$,

which is equivalent to Eq. (13). In order to get a hyperbolic equation, which is a necessary condition for MD causality, Eq. (14) has to be extended. An approach to do this results from an analogy to the classical transmission line. Using this approach one obtains

$l \mathrm{D}_{t} w+\frac{1}{\eta} w+\mathrm{D}_{x} v=0, \quad l>0$,

where some estimate for $l$ will have to be used. Due to the fact that viscosity is now modeled in an extended (and pysically not unrealistic) way, $q_{\mathrm{f}}$ is no longer fully equivalent to Eq. (13).

After introducing normalized variables, applying the coordinate transformation (7), (8) and doing some manipulations Eq. (16) is given by

$$
\begin{aligned}
\sqrt{L_{\mathrm{c}}}\left(\mathrm{D}_{1}+\mathrm{D}_{2}\right) \sqrt{L_{\mathrm{c}}} \tilde{w} \\
+\sqrt{L_{\mathrm{c}}}\left(\mathrm{D}_{1}-\mathrm{D}_{2}\right) \sqrt{L_{\mathrm{c}}} \tilde{v}+R \tilde{w}=0
\end{aligned}
$$




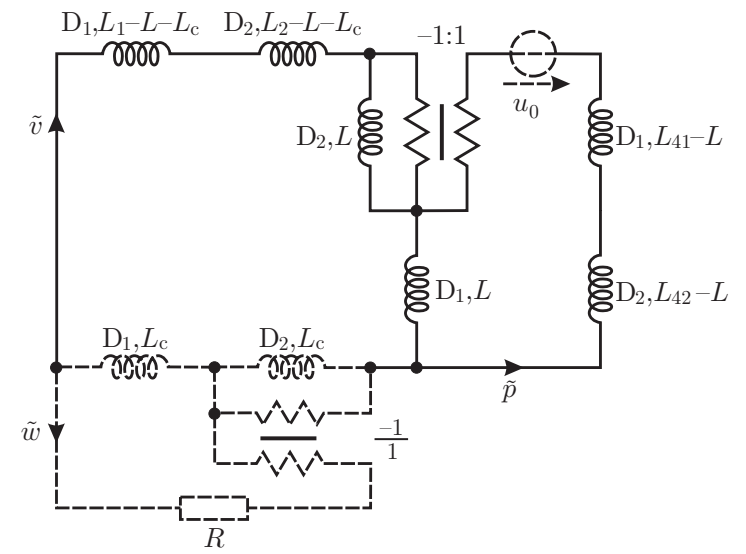

where

$\tilde{w}=l \sqrt{\frac{p}{p_{0}}} w, \quad L_{\mathrm{c}}=\frac{1}{2 l p}, \quad R=\frac{1}{\eta l^{2} p \sqrt{2}}$.

Also this equation can be interpreted as a mesh equation of an appropriate MD Kirchhoff network. Equation (12) can be written in the form

$\tilde{f}=-\sqrt{L_{\mathrm{c}}}\left(\mathrm{D}_{1}+\mathrm{D}_{2}\right) \sqrt{L_{\mathrm{c}}} \tilde{w}$,

and $\tilde{q}_{\text {f results from }}$

$\tilde{q}_{\mathrm{f}}=R \tilde{w}^{2}$,

which corresponds to the energy dissipated in Resistance $R$. At this point all equations are known in order to get two resulting MD Kirchhoff networks which are shown in Fig. 1. At first glance they seem to be independent. However they are implicitly coupled by the inductances which depend on the variables $\rho, p, v$. The dashed part of the network represents the terms of viscosity. In the case that viscosity is not taken into account this part has to be shorted. The energy density $q_{\mathrm{f}}$ is introduced into the network by the represented voltage source where $u_{0}=\frac{\tilde{q}_{\mathrm{f}}}{\tilde{p}}$.

After introducing wave variables and applying the trapezoidal rule a signal flow diagram can be derived. In a further step this can be realized on a computer.

\section{Simulation results}

The Shock Tube Problem is a classical benchmark problem for numerical algorithms in fluid dynamics. It consists of an infinitely large tube which is filled with gas and separated by a diaphragm. The state of the gas on the left side of the diaphragm is different from that on the right side. This results in a discontinous initial distribution. In this paper this distribution is chosen as specified in Hirsch (1990). With this, one obtains

$$
\begin{array}{lll}
\rho_{\mathrm{l}}=1.0 \quad, p_{1}=1.0 & , v_{\mathrm{l}}=0.0, \\
\rho_{\mathrm{r}}=0.125 & , p_{\mathrm{r}}=0.1, & v_{\mathrm{r}}=0.0,
\end{array}
$$

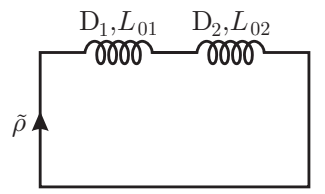

Fig. 1. MD Kirchhoff network which represents the equations of fluid dynamics. for the left and right side of the tube respectively. The isentropic coefficient which has been introduced in Eq. (4) has been chosen $\gamma=1.4$. At $t=0$ the diaphragm is removed and the gas propagates in the tube. According to the Eulerequations during this propagation two discontinuities called shock and contact discontinuity arise. The analytical solution of this problem is given by Hirsch (1990). The Figs. 2 and 3 show the results for pressure, velocity and density at $t=0.14$. Where the analytical solution is plotted with dashed lines and the solution of the Wave Digital Method is plotted with solid lines.

First of all the results without taking into account viscosity are shown in Fig. 2. Similar as in Fries (1994) strong oscillations near the discontinuities occur. Apart from that the curves approximately correspond to the analytical solution.

Whereas in Fig. 3 viscosity terms are taken into account. The friction coefficient $\eta$ is chosen 0.0001 and $l$ is chosen 1 . Near the shock no oscillations occur, because the unsteady curve has changed to a steady curve, which better corresponds to reality. For this the discretization has been chosen very fine. However the oscillations near the contact discontinuity still occur. This is due to the fact that the velocity is constant in this area. Thus the viscosity terms cannot have any influence here.

\section{Conclusions}

The computed results show that the MD Kirchhoff network which represents the Euler-equations can be successfully extended. The derived network takes viscosity into account and represents the Navier-Stokes-equations. Using this it has been shown that the computation of the Shock Tube Problem leads to quite good results. Especially the oscillations near the shock have been suppressed. However a very fine discretisation is required in order to get very steep gradients near the shock. Near the contact discontinuity these oscillations still occur. 

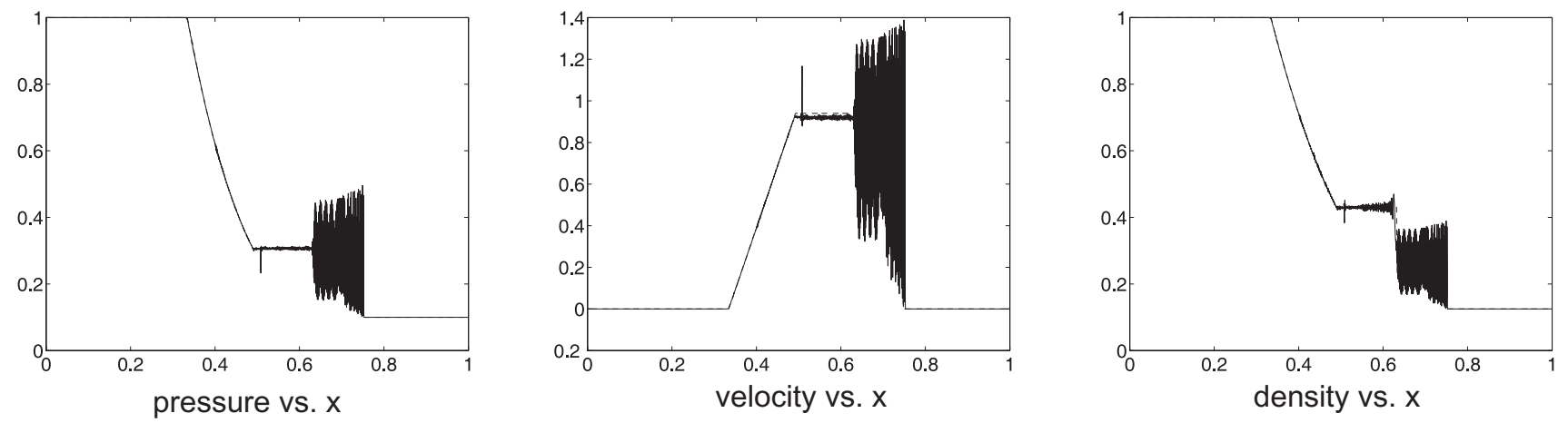

Fig. 2. Numerical Results without taking viscosity into account.
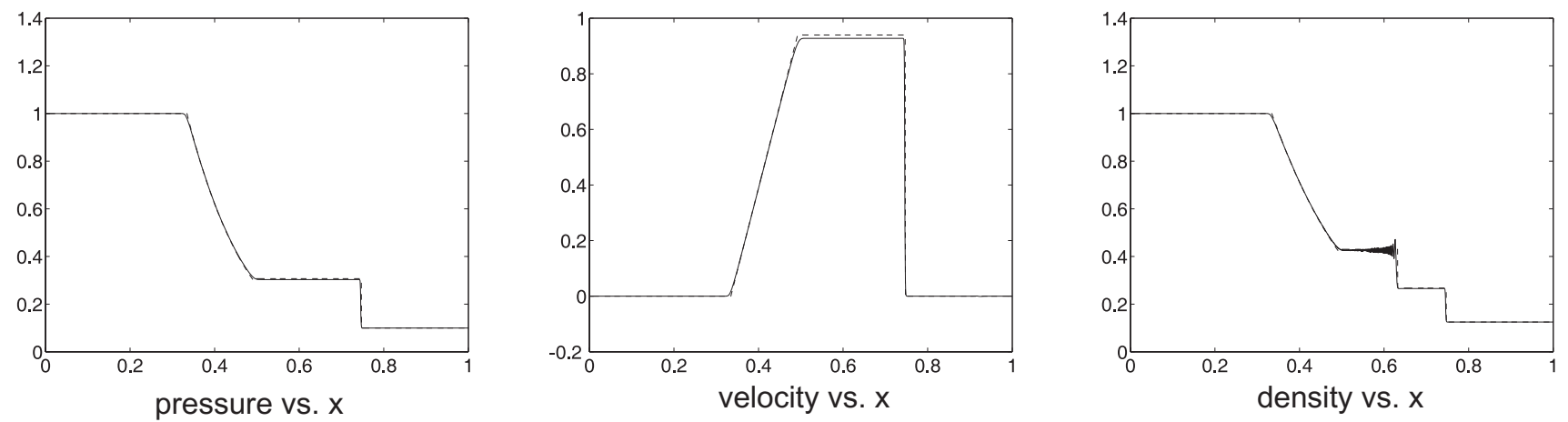

Fig. 3. Numerical Results taking viscosity into account.

Probably taking into account other physical phenomena as for example heat conduction, will result in steady solutions. Using this, oscillations near contact discontinuities could also be suppressed. On the other hand there are many problems in fluid dynamics which do not contain contact discontinuities. So in all probability the Wave Digital Method can be applied successfully to these problems.

\section{References}

Böhme, G.: Strömungsmechanik nichtnewtonscher Fluide, Teubner, 2000.

Fettweis, A.: Wave Digital Filters:Theory and Practice, Proc. IEEE, 74, 270-327, 1986.
Fettweis, A.: Numerical Integration of Partial Differential Equations using Wave-Digital Principles, collection of detailed viewgraphs, Bochum, 2000-2002.

Fettweis, A.: Improved Wave-Digital Approach to numerically Integrating the PDEs of Fluid Dynamics, IEEE ISCAS Proc., Scottsdale, 361-364, 2002.

Fries, M.: Simulation of one-dimensional Euler-flow by means of multidimensional Wave Digital Filters, IEEE ISCAS Proc., London, 6, 9-12, 1994.

Hirsch, C.: Numerical Computation of Internal and External Flows, I, John Wiley and Sons, 1990.

Zhu, Y.: The wave digital PDE simulation method:local passivity considerations for nonlinear PDE systems, Thesis, Notre Dame Univ., 2000. 